\title{
Effective scanning method of the NMSSM parameter space
}

\author{
Conny Beskidt" and Wim de Boer ${ }^{\dagger}$ \\ Dept. of Phys., Karlsruhe Inst. for Technology KIT, 76229 Karlsruhe, Germany
}

(Received 17 May 2019; published 9 September 2019)

\begin{abstract}
The next-to-minimal supersymmetric standard model(NMSSM) naturally provides a $125 \mathrm{GeV}$ Higgs boson without the need for large loop corrections from multi-tera electron volt stop quarks. Furthermore, the NMSSM provides an electroweak scale dark matter candidate consistent with all experimental data, like relic density and nonobservation of direct dark matter signals with the present experimental sensitivity. However, more free parameters are introduced in the NMSSM, which are strongly correlated. A simple parameter scan without knowing the correlation matrix is not efficient and can miss significant regions of the parameter space. We introduce a new technique to sample the NMSSM parameter space, which takes into account the correlations. For this, we project the seven-dimensional NMSSM parameter space onto the three-dimensional Higgs boson mass parameter space. The reduced dimensionality allows for a nonrandom sampling and therefore a complete coverage of the allowed NMSSM parameters. In addition, the parameter correlations and possible deviations of the signal strengths of the observed 125 Higgs boson from the standard model values are easily predicted.
\end{abstract}

DOI: 10.1103/PhysRevD.100.055007

\section{INTRODUCTION}

The next-to-minimal supersymmetric standard model (NMSSM) distinguishes itself from the minimal supersymmetric standard model (MSSM) by a Higgs singlet in addition to the two Higgs doublets of the MSSM. This has three advantages:

(i) The singlet solves the $\mu$ problem, begging the question as to why the dimensionful Higgs mixing parameter $\mu$ in the Lagrangian, which could take any value up to the grand unified theory (GUT) scale, is at the electroweak scale if radiative electroweak symmetry breaking is imposed. In the NMSSM, the $\mu$ parameter is related to the vacuum expectation value (vev) of the singlet, so it is naturally of the order of the electroweak scale; see, e.g., Refs. [1,2].

(ii) The Higgs boson mass at tree level has contributions from the mixing with the singlet, so it is not restricted to be below the $\mathrm{Z}$ mass at tree level, as is the case in the MSSM. Therefore, the NMSSM does not need the large loop corrections from stop quarks to bridge the gap between the $\mathrm{Z}$ mass and the observed Higgs boson with a mass of $125 \mathrm{GeV}[3,4]$. Bridging this gap requires multi-tera electron volt

\footnotetext{
*ConnyBeskidt@kit.edu

†Wim.de.Boer@kit.edu
}

Published by the American Physical Society under the terms of the Creative Commons Attribution 4.0 International license. Further distribution of this work must maintain attribution to the author(s) and the published article's title, journal citation, and DOI. Funded by SCOAP. stop masses in the MSSM; see, e.g., Refs. [5-8] and references therein. However, in the NMSSM, stop-quark masses can be of the order of the teraelectron-volt scale, see, e.g., Ref. [9], so the quadratic divergencies to the Higgs mass are effectively canceled by a not-too-large mass difference between top- and stop-quark masses, thus avoiding the finetuning problem [10-12].

(iii) The dark matter candidate in the NMSSM is usually singlinolike with a mass at the electroweak scale, which fulfills all experimental constraints; especially, it has a direct scattering cross sections with nuclei not yet excluded by experiments (see, e.g., Ref. [13] and references therein).

The introduction of an additional Higgs singlet in the NMSSM yields more parameters for the interactions between the singlet and the Higgs doublets and the singlet self-interaction. One usually performs random scans of the parameters to investigate experimental signatures in the parameter region allowed by the experimental constraints of the observed $125 \mathrm{GeV}$ Higgs boson and its SM-like couplings and/or constraints from the dark matter sector [14-32]. In random scans of highly correlated parameters, it is difficult to reach all parameter combinations ("complete coverage"), since the correlations require simultaneously specific values of several parameters. Such combinations can be found efficiently in random scans only, if a correlation matrix is used to tell in which direction one has to step for parameter $\mathrm{n} 1$, if parameters $\mathrm{n} 2$ to $\mathrm{nx}$ take specific values. Incomplete coverage can lead to wrong predictions, e.g., of the allowed cross sections for spin-dependent and spinindependent direct dark matter searches [13]. To cope with 
the large NMSSM parameter space of the Higgs sector, and especially the large correlations between these parameters, we describe in this paper in detail a novel sampling technique to obtain the allowed range of the NMSSM parameters for the constraints from the observed Higgs boson mass and its couplings. This method was previously used for the analysis of the heavy Higgs boson [33], a light singletlike Higgs boson [34], and dark matter constraints [13] but has never been described in detail.

After a short summary of the Higgs sector in the NMSSM in Sec. II, we present the novel sampling technique to sample the NMSSM parameter space efficiently in Sec. III. In Sec. IV, some applications of the novel sampling technique are presented, like determining the optimal values of the couplings and possible deviations of the signal strengths of the observed $125 \mathrm{GeV}$ Higgs boson from the expected SM-like signal strengths. SM-like signals are only expected for cross sections without loops, since in the loops of gluon fusion and the Higgs boson decays into photons, supersymmetry (SUSY) contributions from, e.g., stop loops may contribute. So, only the signal strengths from diagrams without loops are required to be SM-like, while the possible deviations from diagrams including loops are studied as a function of the stop mass. We restrict the analysis to the well-motivated subspace of the SUSY parameters, using the unification of masses and couplings at the GUT scale and allowing for radiative electroweak symmetry breaking. Present limits on the SUSY masses indicate they are rather heavy and for heavy SUSY masses the Higgs and the SUSY sector largely decouple, except for the stop sector, which influences the $125 \mathrm{GeV}$ Higgs mass and the signal strengths for loopinduced processes. The restriction of the SUSY particle masses by GUT scale parameters does not really restrict the validity of the analysis, since it does not matter if one parametrizes the stop mass with the common mass parameters at the GUT scale or chooses the stop mass directly. However, the GUT scale definition of the parameters has the advantage that the fixed-point solutions of the trilinear couplings are taken into account, thus avoiding values not allowed by solutions of the renormalization group equations (RGEs), as will be discussed later.

\section{HIGGS SECTOR IN THE SEMICONSTRAINED NMSSM}

Within the NMSSM, the Higgs fields consist of the usual two Higgs doublets $\left(H_{u}, H_{d}\right)$ with an additional complex Higgs singlet $S$. The latter singlet distinguishes the NMSSM from the MSSM. The neutral components of the two Higgs doublets and singlet mix to form three physical $C P$-even scalar bosons and two physical $C P$-odd pseudoscalar bosons.

The mass eigenstates of the neutral Higgs bosons are determined by the diagonalization of the mass matrix, see, e.g., Ref. [1], so the scalar Higgs bosons $H_{i}$ are mixtures of the $C P$-even weak eigenstates $H_{d}, H_{u}$ and the singlet $S$,

$$
H_{i}=S_{i 1} H_{d}+S_{i 2} H_{u}+S_{i 3} S,
$$

where the index $i$ increases with the increasing mass of the Higgs boson $H_{i}$ and $S_{i j}$ with $i, j=1,2,3$ are the elements of the $3 \times 3$ Higgs mixing matrix.

As mentioned before, the analysis is restricted to the well-motivated subspace of the SUSY parameters using the unification of masses and couplings at the GUT scale. In contrast to the constrained MSSM, the Higgs mixing parameter $\mu$ is not fixed by radiative electroweak symmetry breaking but is related to the vev of the Higgs singlet and is considered to be a free parameter $\mu_{\text {eff }}$. In total, this semiconstrained NMSSM has nine free parameters:

$$
m_{0}, m_{1 / 2}, A_{0}, \tan \beta, \lambda, \kappa, A_{\lambda}, A_{\kappa}, \mu_{\mathrm{eff}} .
$$

The latter six parameters in Eq. (2) enter the Higgs mixing matrix, thus forming the six-dimensional parameter space of the NMSSM Higgs sector. Here, $\tan \beta$ corresponds to the ratio of the vevs of the Higgs doublets, i.e., $\tan \beta=v_{u} / v_{d}$. The coupling $\lambda$ represents the coupling between the Higgs singlet and doublets, while $\kappa$ corresponds to the self-coupling of the singlet. $A_{\lambda}$ and $A_{\kappa}$ are the corresponding trilinear soft breaking terms. $\mu_{\text {eff }}$ is related to the vev of the singlet $s$ via the coupling $\lambda$, i.e., $\mu_{\text {eff }}=\lambda \cdot s$. Therefore, $\mu_{\text {eff }}$ is naturally of the order of the electroweak scale, thus avoiding the $\mu$ problem; see, e.g., Ref. [2].

In addition, we have the GUT scale parameters of the constrained minimal supersymmetric standard model (CMSSM) $m_{0}, m_{1 / 2}$, and $A_{0}$, where $m_{0}$ and $m_{1 / 2}$ are the common mass scales of the spin 0 and $1 / 2$ SUSY particles at the GUT scale. $A_{0}$ is the trilinear coupling of the CMSSM at the GUT scale. The trilinear coupling $A_{0}$ is highly correlated with $A_{\lambda}$ and $A_{\kappa}$ in the semiconstrained NMSSM, so fixing this parameter would restrict the range of $A_{\lambda}$ and $A_{\kappa}$ severely. Therefore, $A_{0}$ is allowed to vary, which leads to seven free parameters in total and thus a seven-dimensional (7D) NMSSM parameter space. From the free parameters in Eq. (2), the complete SUSY spectrum and all Higgs boson masses can be calculated using the publicly available code NMSSMToOLs [35]. The values of $m_{0}$ and $m_{1 / 2}$ can be fixed to values, which are consistent with the current LHC limits [36]. In the following, we use $m_{0}=m_{1 / 2}=1 \mathrm{TeV}$. The impact of higher common SUSY masses will be discussed in Sec. IV.

One of the lightest NMSSM Higgs bosons should be SMlike. The cross section errors have a significant theoretical error from the dependence on the renormalization - and factorization scales, see, e.g., Ref. [37] and references therein, which can be reduced by using cross section ratios of the NMSSM and SM cross sections, the so-called reduced cross sections. If multiplied by the branching ratios, one obtains the signal strength $\mu_{j}^{i}$ defined as

$$
\mu_{j}^{i}=\frac{\sigma_{i} \times B R_{j}}{\left(\sigma_{i} \times B R_{j}\right)_{\mathrm{SM}}}=c_{i}^{2} \cdot \frac{B R_{j}}{\left(B R_{j}\right)_{\mathrm{SM}}},
$$



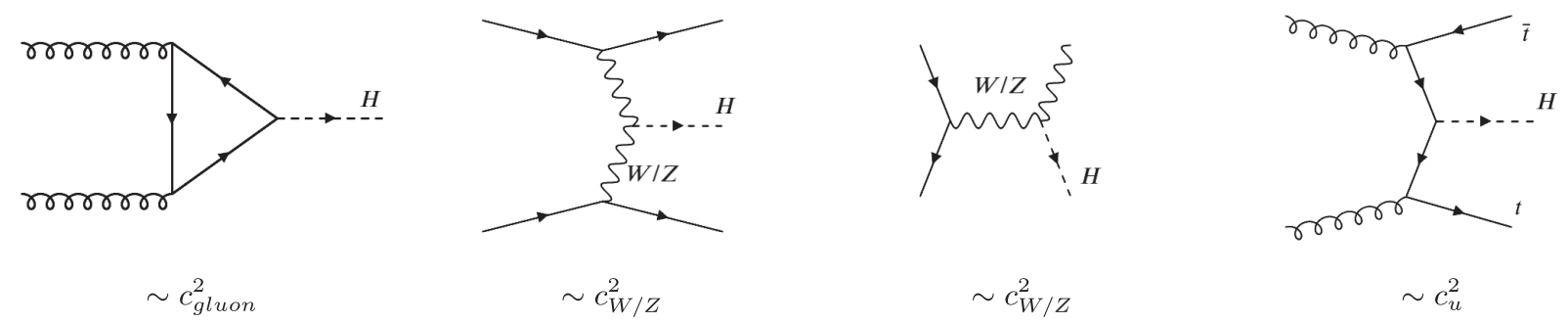

FIG. 1. Summary for the Higgs production channels at the LHC and the corresponding reduced couplings. From left to right: the effective reduced gluon coupling $c_{\text {gluon }}$ for ggf, $c_{W / Z}$ for $\mathrm{VBF}$ and $\mathrm{VH}$, and $c_{u}$ for th.

where the reduced coupling $c_{i}$ squared equals the reduced cross section for production mode $i$, which is multiplied by the corresponding ratio of branching ratios for the decay $j$. The reduced couplings $c_{i}$ depend only on the Higgs mixing matrix elements and $\tan \beta$ for processes without loops. The reduced couplings including loops can have additional contributions from SUSY particles in the loops, preferentially from particles with large couplings to the Higgs boson, like the stop particles. These modify the reduced couplings of Higgs bosons to gluons $c_{\text {gluon }}$ and gammas $c_{\gamma}$, which are parametrized as effective couplings within NMSSMTOOLS.

The diagrams for the used reduced couplings have been summarized in Fig. 1, which shows from left to right the effective reduced gluon coupling $c_{\text {gluon }}$ for gluon fusion (ggf), $c_{W / Z}$ for vector boson fusion (VBF) and Higgsstrahlung (VH), and $c_{u}$ for top fusion (tth). We consider in our analysis two different fermionic final states (b-quarks and $\tau$-leptons) and two different bosonic final states $(W / Z$ and $\gamma$ ) for two different production modes (ggf and VBF) leading to a total of eight reduced cross sections (all calculated in NMSSMTOOLS), which can be divided into signal strengths without (with) effective couplings $\mu_{\text {no-loop }}\left(\mu_{\text {loop }}\right)$ :

$$
\begin{aligned}
\mu_{\text {no-loop }}: & \mu_{\tau \tau}^{V B F / V H}, \quad \mu_{Z Z / W W}^{V B F / V H}, \quad \mu_{b b}^{V B F / V H}, \quad \mu_{b b}^{t t h}, \\
\mu_{\text {loop }}: & \mu_{\tau \tau}^{g g f}, \quad \mu_{Z Z / W W}^{g g f}, \quad \mu_{\gamma \gamma}^{V B F / V H}, \quad \mu_{\gamma \gamma}^{g g f} .
\end{aligned}
$$

The signal strengths $\mu_{\text {loop }}$ include loop diagrams at lowest order, see, e.g., the left diagram in Fig. 1, so SUSY particles in the loop can lead to deviations from the SM prediction. Therefore, it is reasonable to constrain only the signal strengths without loop contributions $\mu_{\text {no-loop }}$ to its SM-like expectation, i.e., $\mu_{\text {no-loop }}=1$, while $\mu_{\text {loop }}$ is allowed to deviate from 1 . So, we impose the following four constraints: $\mu_{\tau \tau}^{V B F / V H}=1, \mu_{b b}^{V B F / V H}=1$, $\mu_{Z Z / W W}^{V B F / V H}=1$, and $\mu_{b b}^{t t h}=1$. The signal strengths $\mu_{\text {loop }}$ can be calculated from the fitted NMSSM parameters using the constraint $\mu_{\text {no-loop }}=1$. The fit will be discussed in the next section.

\section{SAMPLING TECHNIQUE}

The NMSSM parameters in Eq. (2) completely determine the masses of the six Higgs bosons: three scalar Higgs masses $m_{H_{i}}$, two pseudoscalar Higgs masses $m_{A_{i}}$, and the charged Higgs boson mass $m_{H^{ \pm}}$. The masses of $A_{2}, H_{3}$, and $H^{ \pm}$are approximately equal in the decoupling limit, i.e., the mass region with heavy Higgs masses much larger than the Z-boson mass.[38,39] Then, only one of the heavy Higgs masses is independent, which leads in total to a fourdimensional Higgs mass space. Furthermore, one of the masses has to be $125 \mathrm{GeV}$, so only three Higgs masses are free in the decoupling limit, which can be chosen to be $m_{A_{1}}$, $m_{H_{1}}$, and $m_{H_{3}}$. The Higgs masses can be calculated from the NMSSM parameters in Eq. (2), but vice versa each combination of the three masses $m_{A_{1}}, m_{H_{1}}$, and $m_{H_{3}}$ uniquely determines the seven NMSSM parameters assuming the decoupling limit. The parameters can be obtained for each combination of Higgs masses in the threedimensional (3D) space of Higgs masses from a fit to the masses with the NMSSM parameters as free parameters. The fit leads to unique solutions, as will be shown later. The procedure is illustrated in Fig. 2. The fit can be performed for each cell of the 3D Higgs mass space on the left, thus providing for each Higgs mass combination the seven NMSSM parameters on the right panel of Fig. 2. For each set of these parameters, the Higgs mixing matrix is fully specified, and hence the Higgs sector including

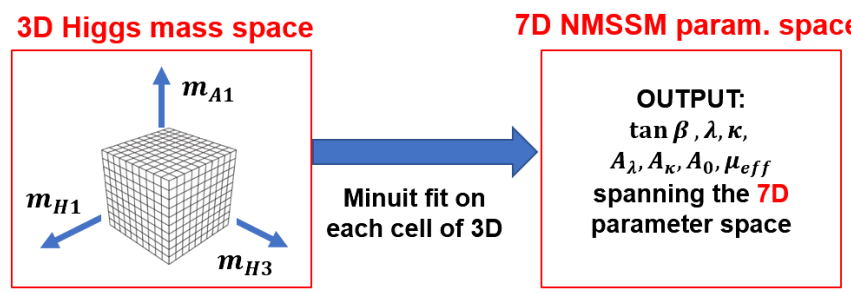

FIG. 2. Sketch of the sampling technique to determine the allowed NMSSM parameter space: Perform a MiNUIT fit on each cell of the 3D Higgs mass space (left box) to determine the corresponding seven free NMSSM parameters (right box). The relation between the NMSSM parameters and the Higgs masses is encoded in NMSSMTOOLS. The second-lightest Higgs boson is chosen to be the $125 \mathrm{GeV}$ Higgs, but we repeat the fit in case $m_{H_{1}}=125 \mathrm{GeV}$. Then, the $m_{H_{1}}$ becomes an $m_{H_{2}}$ axis in the grid on the left. 
masses, couplings, branching ratios, and cross sections can be calculated from these seven parameters.

Note that with the low dimensionality of the 3D grid spanning the Higgs mass space one can perform the fit for each cell in the 3D Higgs mass space, thus providing a complete coverage of the Higgs sector and the corresponding NMSSM parameters without having to resort to a random scan.

For a statistic for the fit determining the NMSSM parameters from the Higgs masses, we choose the $\chi^{2}$ function, which can be minimized by MinuiT [40]. The following contributions are included in the $\chi^{2}$ function,

$$
\chi_{\mathrm{tot}}^{2}=\chi_{H_{S}}^{2}+\chi_{H_{3}}^{2}+\chi_{A_{1}}^{2}+\chi_{H_{125}}^{2}+\chi_{\mu_{125}}^{2}+\chi_{\mathrm{LEP}}^{2}+\chi_{\mathrm{LHC}}^{2},
$$

which are defined as:

(i) $\chi_{H_{S}}^{2}=\left(m_{H_{S}}-m_{\text {grid } H_{S}}\right)^{2} / \sigma_{H_{S}}^{2}$ : The term $\chi_{H_{S}}^{2}$ requires the NMSSM parameters to be adjusted such that the mass of the singletlike light Higgs boson mass $m_{H_{S}}$ agrees with the chosen point in the 3D mass space $m_{\text {grid, } H_{S}}$. The value of $\sigma_{H_{S}}$ is set to 1 per milli of $m_{\text {grid, } H_{S}}$. A small error on the chosen Higgs mass avoids a smearing in the 3D Higgs mass space and a corresponding smearing by the projection onto the 7D parameter space.

(ii) $\chi_{H_{3}}^{2}=\left(m_{H_{3}}-m_{\text {grid, } H_{3}}\right)^{2} / \sigma_{H_{3}}^{2}$ : As $\chi_{H_{S}}^{2}$, but for the heavy scalar Higgs boson $\mathrm{H}_{3}$.

(iii) $\chi_{A_{1}}^{2}=\left(m_{A_{1}}-m_{\text {grid }, A_{1}}\right)^{2} / \sigma_{A_{1}}^{2}$ : As $\chi_{H_{S}}^{2}$, but for the light pseudoscalar Higgs boson $A_{1}$.

(iv) $\chi_{H_{125}}^{2}=\left(m_{H_{125}}-m_{\mathrm{obs}}\right)^{2} / \sigma_{125}^{2}$ : This term is analogous to the term for $m_{H_{S}}$, except that the Higgs mass $m_{H_{125}}$ is required to agree with the observed Higgs boson mass, so $m_{\mathrm{obs}}$ is set to $125.2 \mathrm{GeV}$. The corresponding uncertainty $\sigma_{125}$ was set to 1 per milli of $m_{\mathrm{obs}}$. Note that the much larger error on the mass of the observed $125 \mathrm{GeV}$ boson is not taken into account, since we want to determine the NMSSM parameters for a precise scan of the Higgs mass parameter space. Once the NMSSM parameters have been determined, one can look for the region where the predicted Higgs mass is within the experimental errors of the observed Higgs mass.

(v) $\chi_{\mu_{125}}^{2}=\sum_{i}\left(\mu_{H_{125}}^{i}-\mu_{\text {theo }}\right)^{2} / \sigma_{\mu}^{2}$ : This term requires the Higgs boson $H_{125}$ to have SM-like couplings for the four signal strengths, labeled as $\mu_{\text {no-loop }}$ in Eq. (4), so $\mu_{\text {theo }}=1$, and $\sigma_{\mu}$ was chosen to be 0.005 . The remaining four reduced cross sections $\mu_{\text {loop}}$, which include either gluons and/or gammas, can be calculated from the fitted NMSSM parameters and are allowed to deviate from one because of the SUSY contributions, which depend on the choice of $m_{0}, m_{1 / 2}$.

(vi) LEP constraints: $\chi_{\text {LEP }}^{2}$ includes the LEP constraints on the couplings of a light Higgs boson below
$115 \mathrm{GeV}$ and the limit on the chargino mass. These constraints include upper limits on the decay of light Higgs bosons into b-quark pairs, which are particularly important for the singlet Higgs, if it is the lightest one. The LEP constraints are in principle implemented in NMSSMTOOLS, but small corrections were applied, as discussed in Ref. [41].

(vii) LHC constraints: $\chi_{\text {LHC }}^{2}$ includes constraints from the LHC, as implemented in NMSSMToOLS, concerning light scalar and pseudoscalar Higgs bosons. [42-44] We assume that the constraints on SUSY mass limits from LEP and LHC as well as the Higgs masses are uncorrelated. Note that we either assume the lightest Higgs $H_{1}$ to be the singletlike Higgs boson $H_{S}$ and the second lightest Higgs boson $H_{2}$ to be the $125 \mathrm{GeV} \mathrm{SM} \mathrm{Higgs} \mathrm{boson} H_{\mathrm{SM}}$ or vice versa. In the first case, the singletlike Higgs boson has a mass below $125 \mathrm{GeV}$, while for the second case, the mass is above $125 \mathrm{GeV}$. There are also solutions where $m_{H_{1}}=$ $125 \mathrm{GeV}, m_{\mathrm{H}_{2}}>125 \mathrm{GeV}$, and $m_{\mathrm{H}_{3}}$ is singletlike, but we focus on the scenarios where the heavy Higgs bosons are MSSM-like, which leads naturally to $m_{A_{2}} \approx m_{H_{3}}$ and is a reasonable assumption. We restrict the range of the Higgs masses in the 3D mass space as follows:

$$
\begin{aligned}
5 \mathrm{GeV} & <m_{H_{S}}<500 \mathrm{GeV}, \\
125 \mathrm{GeV} & <m_{H_{3}}<2 \mathrm{TeV}, \\
5 \mathrm{GeV} & <m_{A_{1}}<500 \mathrm{GeV} .
\end{aligned}
$$

Although heavier Higgs bosons are not forbidden, they are not relevant for LHC physics, so we did not investigate them here.

\section{EXEMPLARY RESULTS}

The fit for all Higgs mass combinations in the left box of Fig. 2 leads to the allowed region of the NMSSM parameters in the right box. The left side of Fig. 3 shows the $\chi^{2}$ distribution as function of $\tan \beta$ for $m_{0}=m_{1 / 2}=$ $0.7 / 1.0 / 1.3 \mathrm{TeV}$ from top to bottom, respectively, for a given cell in the left panel of Fig. 2 (in this case $m_{H_{1}}=90 \mathrm{GeV}$, $\left.m_{H_{3}}=1000 \mathrm{GeV}, m_{A_{1}}=200 \mathrm{GeV}\right)$. The main contribution to the $\chi^{2}$ is coming from the signal strengths, which are close to the SM expectation of 1 for a large range of $\tan \beta$ for processes without loops, as shown on the middle panels of Fig. 3. However, the signal strengths including loop contributions deviate from 1 because of the SUSY contributions. The deviations are strongest for light stop masses (top row); the gluon fusion reduced signal strengths decrease by approximately $7 \%$ if the stop mass decreases from 800 to $400 \mathrm{GeV}$ for $\tan \beta$ increasing from 3 to 6 . The signal strength including the decay into gammas increases at the same time by approximately $2 \%$. The stop masses as a function of $\tan \beta$ ) are shown on the panels on the right for the different choices of $m_{0}, m_{1 / 2}=$. Larger SUSY masses decrease the 
contribution from the stop loops, thus reducing the signal strength dependence on $\tan \beta$, as can be seen from the panels in the middle of Fig. 3.

The shift in the minimum of the $\chi^{2}$ function to higher $\tan \beta$ in the middle and bottom rows is caused by the increase in the stop mass, since the reduced stop corrections to the $125 \mathrm{GeV}$ Higgs boson mass can be compensated largely by an increase in $\tan \beta$, although other parameters change somewhat as well for the minimum $\chi^{2}$ value.

Not only a shift but also a broader $\chi^{2}$ distribution is shown for $m_{0}, m_{1 / 2}$ varying from 0.7 to $1.3 \mathrm{TeV}$. This originates mainly from the $125 \mathrm{GeV}$ mass constraint of the observed boson and can be understood as follows: for the lowest stop mass $\left(m_{0}, m_{1 / 2}=0.7 \mathrm{TeV}\right)$, one has the smallest loop corrections to the $125 \mathrm{GeV}$ Higgs mass, and one needs a large mass correction from NMSSM mixing between the singlet and other Higgs bosons, which requires a precise tuning of the NMSSM parameters to reach the value of $125 \mathrm{GeV}$. For a heavier stop mass, the loop corrections to the $125 \mathrm{GeV}$ Higgs boson are larger, and the contribution from mixing with the singlet can be smaller, which leads to more freedom in the NMSSM parameters and thus a broader $\chi^{2}$ distribution. We have checked this by imposing a smaller value below $125 \mathrm{GeV}$ for the observed Higgs boson mass, in which case the freedom in the NMSSM parameters is larger for a given stop mass, thus leading to a broader $\chi^{2}$ distribution. This is demonstrated in Appendix A, where we used hypothetical values of 123 , 124, and $125 \mathrm{GeV}$ for the observed Higgs boson.

The NMSSM parameters are correlated, but they are not degenerate, as can be observed from the correlations between the parameters in the Minuit output. The maximum correlation occurs between the parameters $\mu_{\text {eff }}$ and $\kappa$, but the correlation is still below 0.76, and other correlations are smaller, as shown for a given mass combination in Appendix B. After fitting all cells in the 3D Higgs mass space (left side of Fig. 2), one obtains the allowed regions of the parameters, which are shown for a few parameters in the two-dimensional plots in Fig. 4. The parameters $\tan \beta$ and $\mu_{\text {eff }}$ show a strong negative correlation, while the trilinear couplings show a strong positive correlation for the GUT scale input parameters. The values of the trilinear couplings
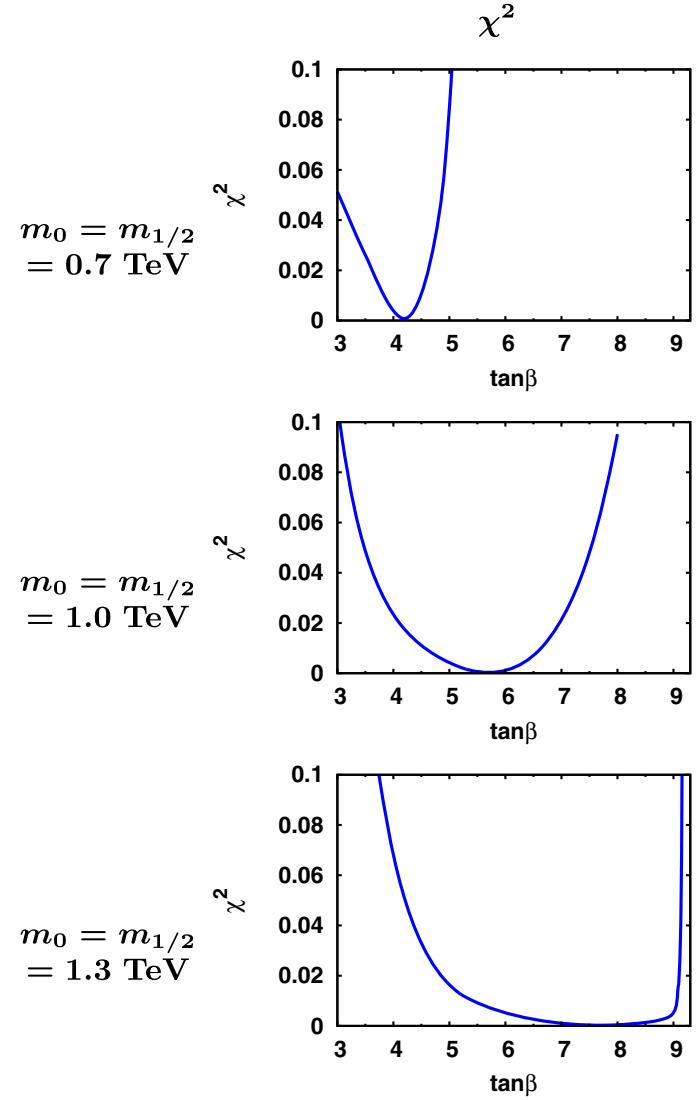
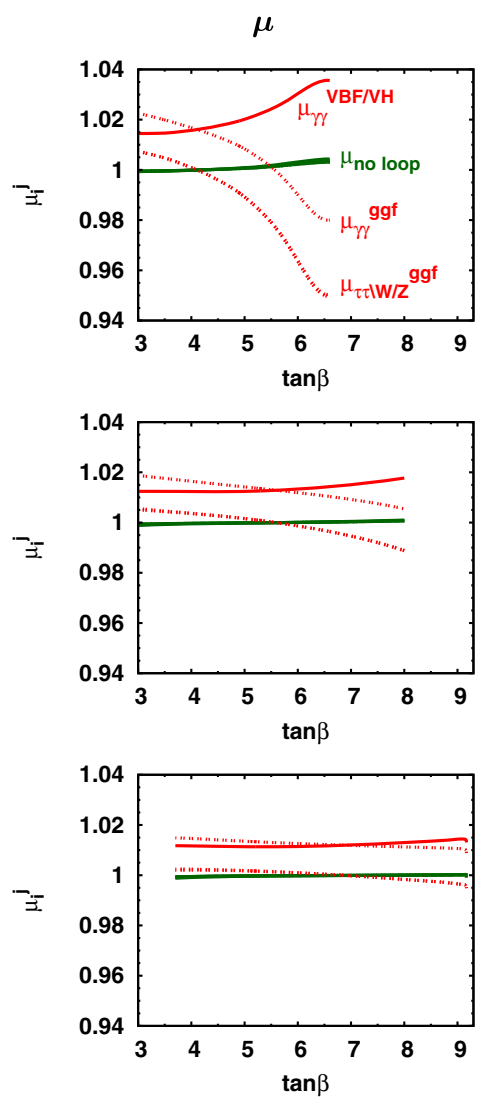
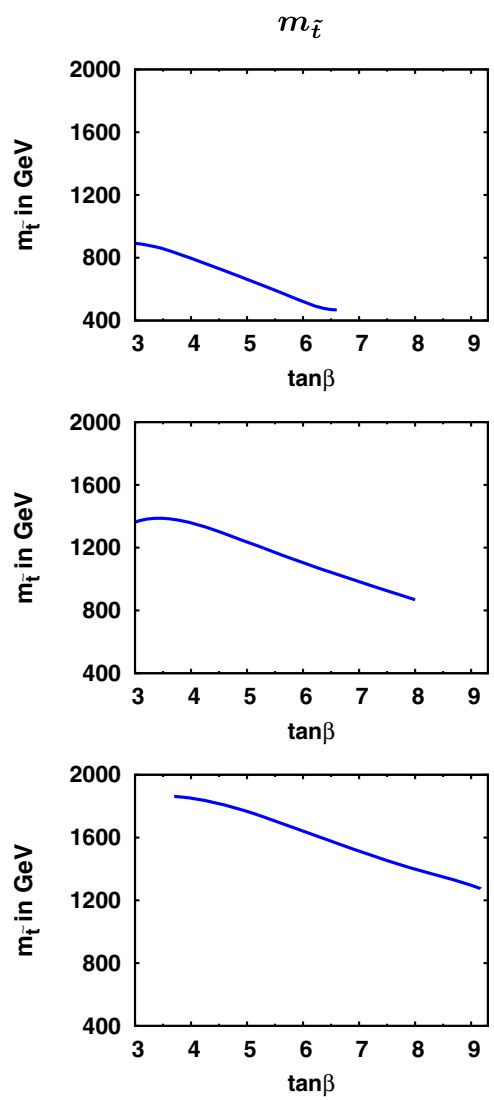

FIG. 3. The left side shows the $\chi^{2}$ distribution as a function of $\tan \beta$ for $m_{0}=m_{1 / 2}=0.7 / 1.0 / 1.3 \mathrm{TeV}$ from top to bottom, respectively. The fits are for an exemplary Higgs mass combination of $m_{H_{1}}=90 \mathrm{GeV}, m_{H_{3}}=1000 \mathrm{GeV}, m_{A_{1}}=200 \mathrm{GeV}$. The main contributions to the $\chi^{2}$ are coming from the signal strengths, which are shown in the middle panels for $m_{0}=m_{1 / 2}=0.7 / 1.0 / 1.3 \mathrm{TeV}$, respectively. The signal strengths including gammas and/or gluons deviate from the SM prediction, since the corresponding reduced couplings, and hence the signal strengths, are sensitive to SUSY contributions. These contributions vary with the mass of the SUSY particles, which vary strongly from top to bottom, as can be seen from the right panels for $m_{0}=m_{1 / 2}=0.7 / 1.0 / 1.3 \mathrm{TeV}$. 

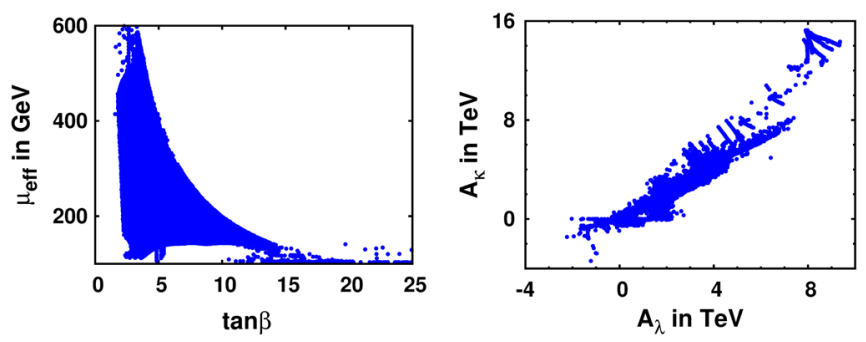

FIG. 4. Allowed ranges for a few NMSSM parameters in the planes $\tan \beta-\mu_{\text {eff }}$ (left) and $A_{\kappa}-A_{\lambda}$ (right).

at the SUSY scale are much more restricted than their values at the GUT scale because of the fixed-point solutions of the RGEs for $A_{\lambda}$ and $A_{\kappa}$, which means that the SUSY scales are largely independent of the GUT scale values. However, the SUSY scale values depend on the chosen Higgs masses, so the ranges of the SUSY scale values of $A_{\lambda}$ and $A_{\kappa}$ are still appreciable, as demonstrated in Appendix C.

The allowed range of $\lambda-\kappa$ depends on the choice of $m_{0}=m_{1 / 2}=1$, as demonstrated in Fig. 5. One can identify two preferred regions, which can be understood if one considers the approximate expression [2] for a 125 GeV Higgs:

$M_{H}^{2} \approx M_{Z}^{2} \cos ^{2} 2 \beta+\Delta_{\tilde{t}}+\lambda^{2} v^{2} \sin ^{2} 2 \beta-\frac{\lambda^{2}}{\kappa^{2}}(\lambda-\kappa \sin 2 \beta)^{2}$.

The first tree level term can become at most $M_{Z}^{2}$ for large $\tan \beta$. The difference between $M_{Z}$ and $125 \mathrm{GeV}$ has to originate mainly from the logarithmic stop mass corrections $\Delta_{\tilde{t}}$ and/or the two remaining terms originating from the mixing between the $125 \mathrm{GeV}$ Higgs boson and the singlet of the NMSSM. The mixing becomes large for large values of the couplings $\lambda$ and $\kappa$ and small $\tan \beta$. Note that the mixing contributes at tree level, so there is no logarithmic dependence, as is the case for the stop loop contribution. The allowed region with large values of the couplings $\lambda$ and $\kappa$ is called Region $I$ in the following. However, there exists a second solution to Eq. (7) with small values of $\lambda, \kappa$ and larger values of $\tan \beta$, which can be obtained by a tradeoff between the first two terms and the last two terms, which we call Region II. Region II with its small couplings $\lambda$ and $\kappa$ is in some sense closer to the MSSM, although the singletlike Higgs and its corresponding singlinolike lightest supersymmetric particle yield additional physics, like the possibility of double Higgs production and a lightest supersymmetric paricle hardly coupling to matter. If the values of $m_{0}$ and $m_{1 / 2}$ are increased, the SUSY contribution to the tree level Higgs mass becomes larger, so the contribution from the mixing with the singlet can be smaller. Then, there is more freedom for the values of the NMSSM parameters and regions I and II start to merge, as can be observed from the right panel in Fig. 5. For larger values of $m_{0}, m_{1 / 2}$, the deviations of the signal values from the SM expectation start to decrease as well, as shown in Appendix D.

Intermediate values for $\lambda$ and $\kappa$ in Fig. 5 are disfavored by the fit because of higher $\chi^{2}$ values, mainly from the signal strengths. This is discussed in more detail in Appendix E.

\section{SUMMARY}

In this paper, a new technique to sample the NMSSM parameter space is introduced and allows an efficient sampling with complete coverage. This is obtained by sampling the 3D Higgs mass space instead of the large 7D NMSSM parameter space. The reduction in the dimensionality is possible by assuming that the heavy Higgs masses $m_{H_{3}}, m_{A_{2}}$, and $m_{H^{ \pm}}$are approximately equal, which is true in the decoupling limit. Furthermore, one of the lighter Higgs bosons has to correspond to the observed $125 \mathrm{GeV}$ Higgs boson. So, instead of the six Higgs boson masses, one has only three independent Higgs masses. This 3D mass space can be subdivided in cells in the $m_{H_{3}}, m_{H_{1}}, m_{A_{1}}$ mass space, as shown in Fig. 2 on the left-hand side. For each cell, the corresponding NMSSM parameters can be determined by a MiNuIT fit to the Higgs masses with the NMSSM parameters as free parameters, a procedure which one would follow if all Higgs masses would have been measured. Here, we assumed that the second lightest boson corresponds to the observed boson, i.e., $m_{\mathrm{H}_{2}}=125 \mathrm{GeV}$. In case $m_{H_{1}}=125 \mathrm{GeV}$, the $3 \mathrm{D}$ mass space would be spanned by $m_{H_{3}}, m_{H_{2}}$, and $m_{A_{1}}$, an option which was investigated as well. The reduced $3 \mathrm{D}$ dimensionality of the Higgs mass space allows one to perform the fit for each cell, so one does not need a random random sampling of the parameter space. As discussed in the Introduction, a random scan of highly correlated parameters (see Fig. 4 for part of the correlations) will need a correlation matrix to
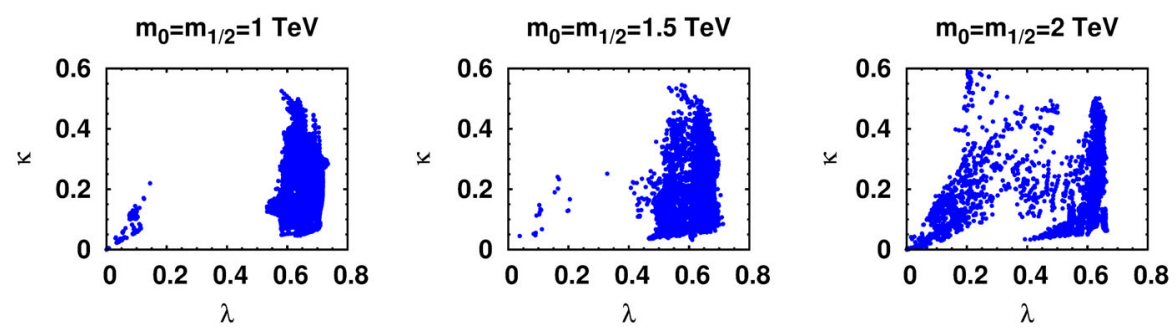

FIG. 5. Allowed range for $\lambda-\kappa$ for $m_{0}=m_{1 / 2}=1,1.5$ and $2 \mathrm{TeV}$, respectively (left to right). 
efficiently scan the parameter space and guarantee complete coverage. For example, one can observe from Fig. 4 immediately that if $\mu_{\text {eff }}$ is scanned up to $1 \mathrm{TeV}$ and $\tan \beta$ is scanned up to 60 many points for random values of $\mu_{\text {eff }}$ and $\tan \beta$ are disfavored by higher $\chi^{2}$ values, since only the indicated points fulfill all requirements and lead to small $\chi^{2}$ values. In the $\lambda, \kappa$ plane, only two regions are preferred: region I (II) at large (small) values of $\lambda, \kappa$, as shown in Fig. 5. Why the $\chi^{2}$ values increase in the intermediate regions is discussed in Appendix E. The size of the regions depends on the values of the SUSY parameters, as is obvious from a comparison of the panels in Fig. 5. This can be understood as follows: the $125 \mathrm{GeV}$ Higgs mass has at tree level contributions from the mixing with the singlet Higgs boson (largely determined by the Higgs couplings $\kappa$, $\lambda$ ) and the stop loops (largely determined by the stop mass). For light stops, the corrections from stop loops are small, and the values of $\lambda, \kappa$ have to be precisely tuned to reach the $125 \mathrm{GeV}$ mass. For larger values of the stop masses, one has more freedom in these NMSSM parameters, and the allowed regions grow and start to overlap.

In summary, the novel scanning technique allows us to study the NMSSM parameter space in a nonrandom way, which guarantees complete coverage and reveals many interesting features, like the high correlations of the NMSSM parameters and possible deviations of the signal strengths from the SM expectation for processes with SUSY particles contributing in the loops.

\section{APPENDIX A: IMPACT OF THE COMMON SUSY MASSES}

The broad $\chi^{2}$ distribution shown in Fig. 3 results from the SM Higgs mass constraint of $125 \mathrm{GeV}$. A smaller Higgs mass requires smaller radiative stop corrections, leading to more freedom in the choice of the NMSSM parameters and hence to a broader $\chi^{2}$ distribution for the same common SUSY masses $m_{0}, m_{1 / 2}$. The same effect is observed if smaller values of the SM Higgs mass are considered for small common SUSY masses. In this case, a larger region of the NMSSM parameter space is compatible with the Higgs boson mass, leading to a

\begin{tabular}{|c|c|c|c|c|c|c|c|}
\hline & $\tan \beta$ & $A_{0}$ & $A_{\kappa}$ & $A_{\lambda}$ & $\lambda$ & $\kappa$ & $\mu_{\text {eff }}$ \\
\hline $\tan \beta$ & ( 1.000 & -0.350 & 0.197 & -0.182 & -0.396 & 0.528 & -0.685 \\
\hline$A_{0}$ & -0.350 & 1.000 & -0.157 & 0.680 & -0.312 & -0.186 & 0.135 \\
\hline$A_{\kappa}$ & 0.197 & -0.157 & 1.000 & 0.345 & 0.218 & 0.306 & -0.225 \\
\hline$A_{\lambda}$ & -0.182 & 0.680 & 0.345 & 1.000 & -0.218 & -0.374 & 0.273 \\
\hline$\lambda$ & -0.396 & -0.312 & 0.218 & -0.218 & 1.000 & -0.406 & 0.385 \\
\hline$\kappa$ & 0.528 & -0.186 & 0.306 & -0.374 & -0.406 & 1.000 & -0.755 \\
\hline$\mu_{\mathrm{eff}}$ & -0.685 & 0.135 & -0.225 & 0.273 & 0.385 & -0.755 & 1.000 \\
\hline
\end{tabular}
$125 \mathrm{GeV}$. while $m_{0}=m_{1 / 2}=0.7 \mathrm{TeV}$. rily in Fig. 4,

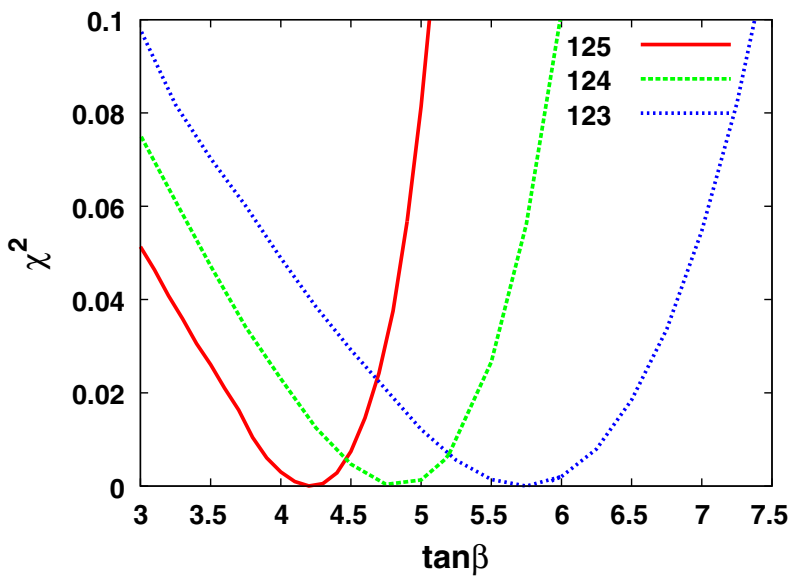

FIG. 6. $\chi^{2}$ distribution for $m_{H_{1}}=90 \mathrm{GeV}, m_{H_{3}}=1000 \mathrm{GeV}$, $m_{A_{1}}=200 \mathrm{GeV}$ and $m_{0}=m_{1 / 2}=0.7 \mathrm{TeV}$. If hypothetically smaller values of the Higgs mass are considered, the $\chi^{2}$ distribution shifts and gets broader because the NMSSM parameters need not to be so finely tuned anymore to reach the

broader $\chi^{2}$ distribution. This is shown in Fig. 6 for $123-125 \mathrm{GeV}$. The Higgs mass combination was chosen to be $m_{H_{1}}=90 \mathrm{GeV}, m_{H_{3}}=1000 \mathrm{GeV}, m_{A_{1}}=200 \mathrm{GeV}$,

\section{APPENDIX B: CORRELATION MATRIX EXAMPLE}

Example of a MinUiT output of the correlation coefficients for the global minimum for the Higgs mass combination $m_{H_{1}}=90 \mathrm{GeV}, m_{H_{3}}=1000 \mathrm{GeV}, m_{A_{1}}=$ $200 \mathrm{GeV}$ and $m_{0}=m_{1 / 2}=1 \mathrm{TeV}$. The highest correlation of 0.755 is observed for the parameters $\kappa$ and $\mu_{\text {eff }}$, but this is still far from degeneracy, so the fit finds welldefined minima for all parameters, as demonstrated in Fig. 3. Note that these are the correlations between the parameter values of a single fit leading to single values of the parameters. If summed over all fits, the fitted parameters vary in a correlated way, as shown exempla- 

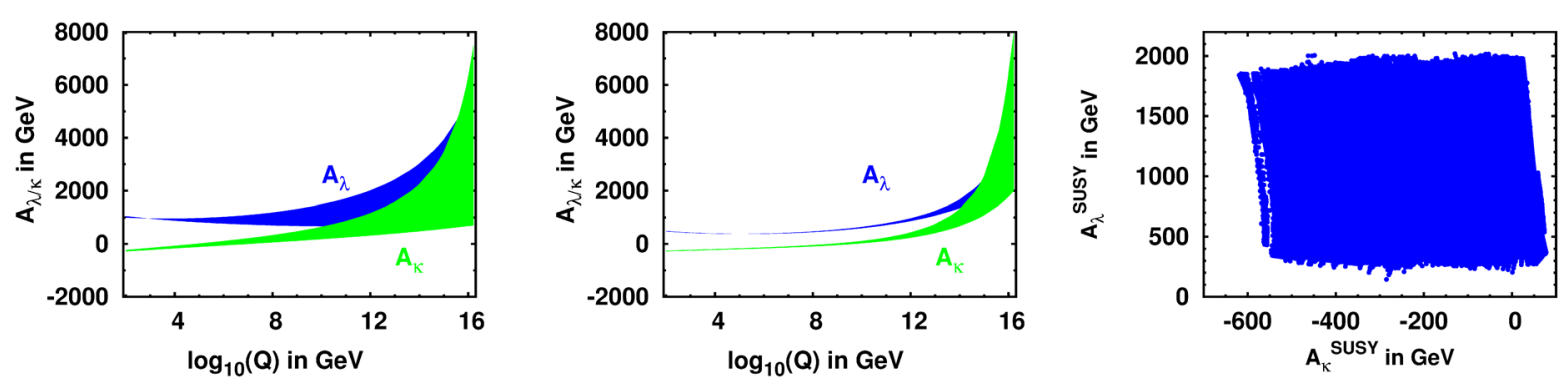

FIG. 7. The left two panels show the running of the trilinear couplings $A_{\lambda}$ (dark shaded (blue) region) and $A_{\kappa}$ (light shaded (red) region) from the GUT to the SUSY scale for two different Higgs mass combinations. One observes a fixed-point solution; i.e., the low energy values are largely independent of the choice of the GUT scale value. By a comparison of the fixed-point solutions in the left and middle panels, one observes that the fixed-point solutions are different; i.e., they depend on the chosen mass combination. If summed over all mass combinations, the spread in the SUSY scale values (the fixed-point solutions) is still not so small, as shown in the panel on the right.

\section{APPENDIX C: RUNNING OF THE TRILINEAR COUPLINGS}

The GUT scale values of the trilinear couplings $A_{\lambda}$ and $A_{\kappa}$ have a large allowed range, as shown in Fig. 4 in the text.
However, the values at the SUSY scale have only small variations, corresponding to the so-called fixed-point solutions from the RGEs, as shown for two mass combinations on the two left panels of Fig. 7. From a comparison of the
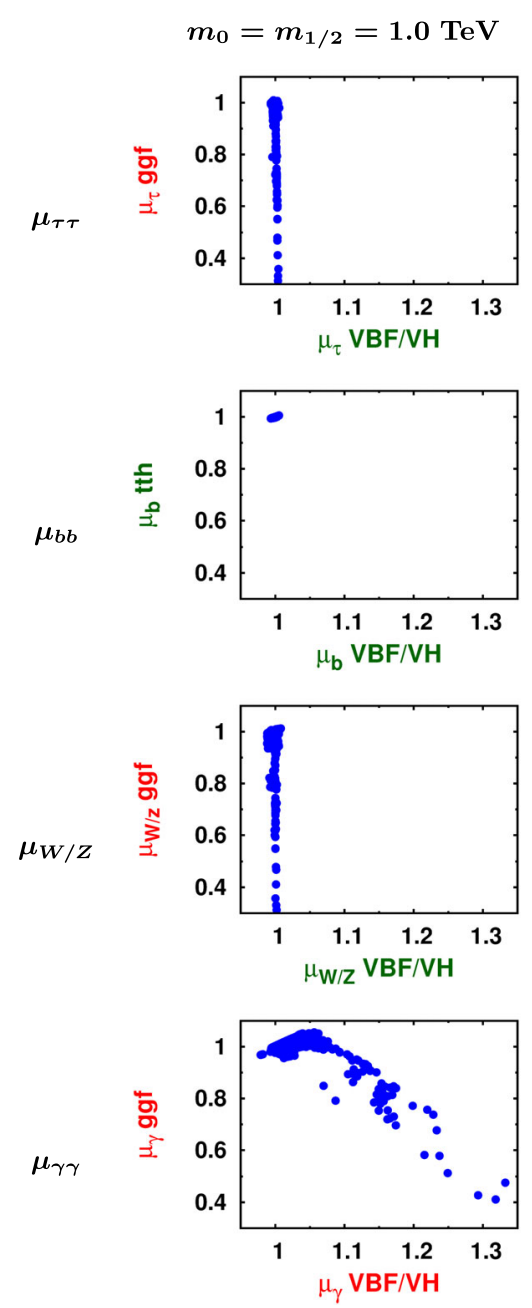
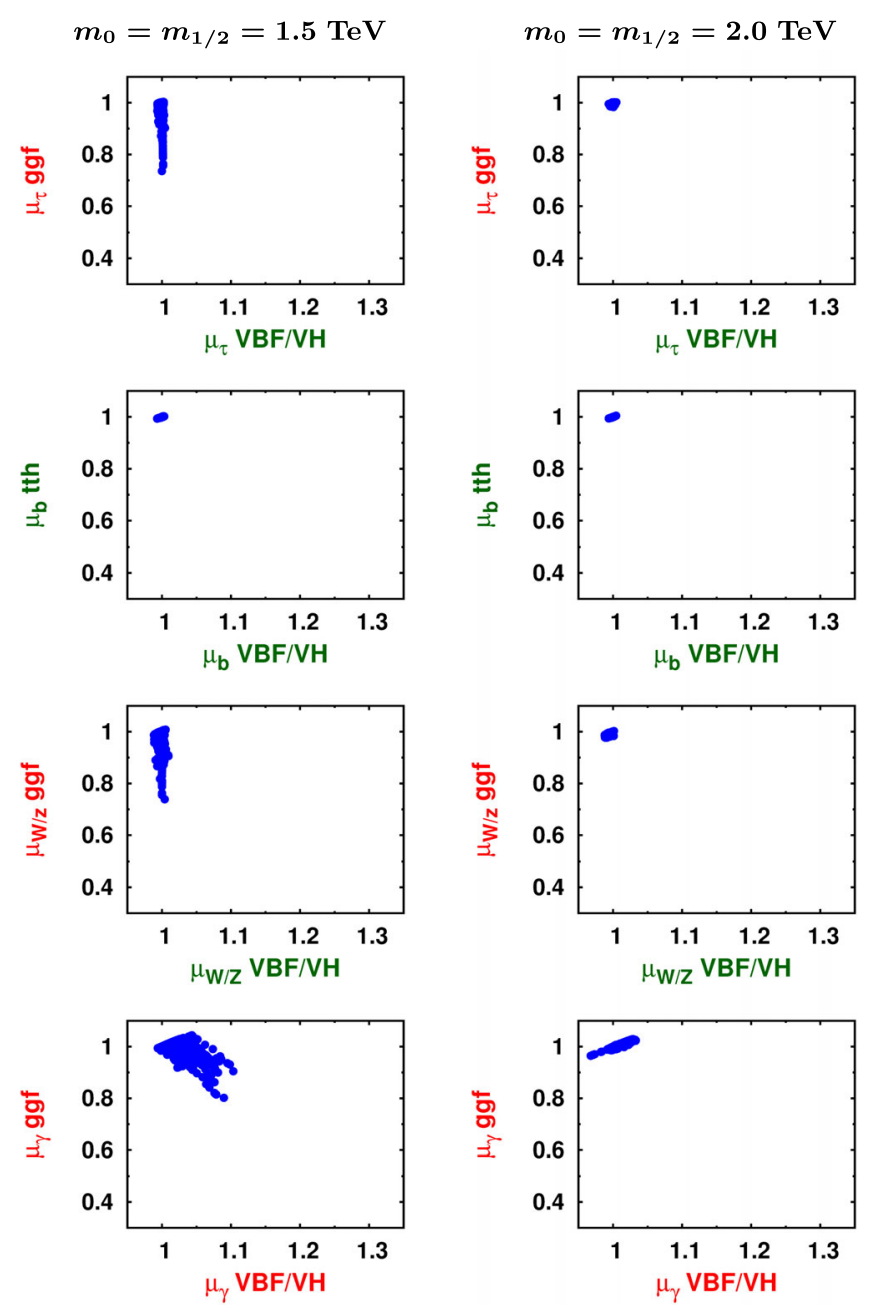

FIG. 8. Allowed ranges for all eight signal strengths from Eq. (4) for $m_{0}=m_{1 / 2}=1,1.5,2 \mathrm{TeV}$, respectively (from left to right). Instead of showing the range in eight one-dimensional histograms, the eight axes are displayed on four two-dimenional plots. The axes labeled in green correspond to signal strengths without loops and are always close to the SM expectation of one, while the axes labeled in red correspond to signal strengths with loops, which can deviate from one, especially for low stop mass (left column) implying large SUSY contributions. 
TABLE I. Differences of the fitting procedures for the standard fit in Sec. III (left column) and the fit parameters for the $\lambda-\kappa$ scan (right column).

\begin{tabular}{lcc}
\hline \hline Procedure & Standard & $\lambda-\kappa$ scan \\
\hline Input & $A_{1}, H_{1 / 2}, H_{3}$ & $\lambda, \kappa$ \\
Constraints & $H_{1 / 2}=125 \mathrm{GeV}$, & $H_{1 / 2}=125 \mathrm{GeV}$, \\
& $\mu_{\text {no-loop }}=1$ & $\mu_{\text {no-loop }}=1$ \\
Output & $\tan \beta, A_{0}, A_{\kappa}$, & $\tan \beta, A_{0}, A_{\kappa}, A_{\lambda}, \mu_{\text {eff }}$ \\
& $A_{\lambda}, \mu_{\text {eff }}, \lambda, \kappa$ & \\
$\chi^{2}$ contribution & $\chi_{H_{S}}^{2}, \chi_{H_{3}}^{2}, \chi_{A_{1}}^{2}$, & $\chi_{H_{\mathrm{SM}}}^{2}, \chi_{\mu_{\mathrm{SM}}}^{2}$, \\
& $\chi_{H_{\mathrm{SM}}}^{2}, \chi_{\mu_{\mathrm{SM}}}^{2}, \chi_{\mathrm{LEP}}^{2}, \chi_{\mathrm{LHC}}^{2}$ & $\chi_{\mathrm{LEP}}^{2}, \chi_{\mathrm{LHC}}^{2}$ \\
\hline \hline
\end{tabular}

two left panels, one observes that the fixed point is different for different Higgs mass combinations, so the SUSY scale values still show variation, if summed over all mass combinations, as shown on the right panel of Fig. 7.

\section{APPENDIX D: SIGNAL STRENGTHS VERSUS SUSY MASSES}

The results for the signal strengths from the Higgs mass sampling is shown in Fig. 8 by the blue dots for $m_{0}, m_{1 / 2}$ increasing from 1 to $2 \mathrm{TeV}$ from left to right. The no-loop (loop) signal strengths are indicated by the green (red) colors on the axes. The fitted no-loop signal strengths are close to 1 , but the signal strengths including loops differ from 1 . One observes deviations of $\mu_{\text {loop }}$ up to $70 \%$, e.g., for $\mu_{\tau}^{g g f}$ for $m_{0}=m_{1 / 2}=1 \mathrm{TeV}$ in the left panel on the top. The tails of the distributed points represent points in the parameter space with low stop masses, which affect the loop signal strengths strongest. Larger common SUSY masses $m_{0}, m_{1 / 2}$ for the panels in the two right columns lead to heavier stops (see Fig. 3) and hence smaller SUSY contributions in the loops, leading to smaller deviations of the signal strengths.

\section{APPENDIX E: RESULTS FROM THE $\lambda-\kappa$ SCAN}

As discussed in Sec. IV, there are two preferred regions in the $\lambda, \kappa$ plane varying in size with the stop mass, as shown in Fig. 5. One wonders what happens outside the preferred regions. In order to find out, the standard fit procedure was slightly modified by requiring the NMSSM parameters $\lambda$ and $\kappa$ to be fixed in the fit. The fit with fixed $\lambda$ and $\kappa$ parameters was repeated for all $\kappa, \lambda$ combinations in the $\lambda-\kappa$ plane and check which constraints increase the $\chi^{2}$ of the fit outside the preferred regions. Fixing the $\kappa$, $\lambda$ values in the fit reduces the number of free parameters to only 5 , namely $\tan \beta, A_{0}, A_{\kappa}, A_{\lambda}$, and $\mu_{\text {eff }}$. The constraints are reduced, too, since the free Higgs boson masses $A_{1}, H_{1}$, and $H_{3}$ are allowed to vary to minimize

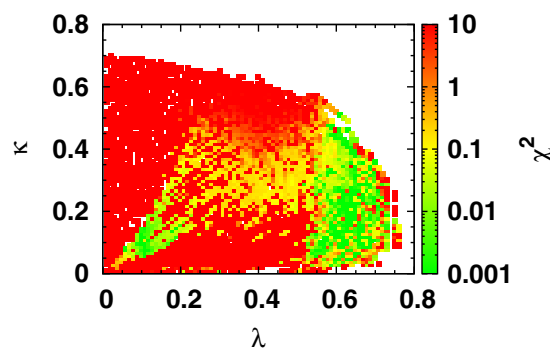

(a)

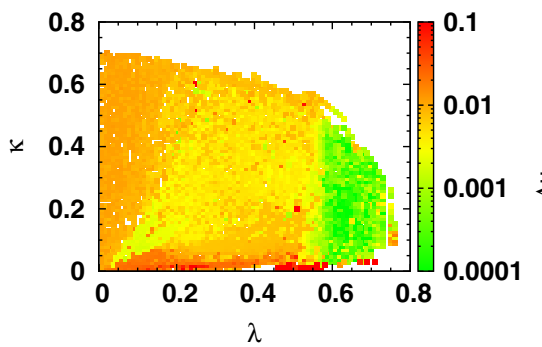

(c)

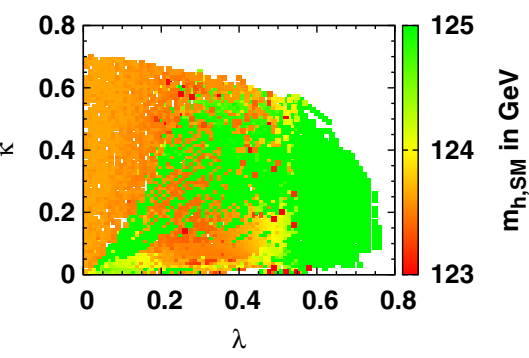

(b)

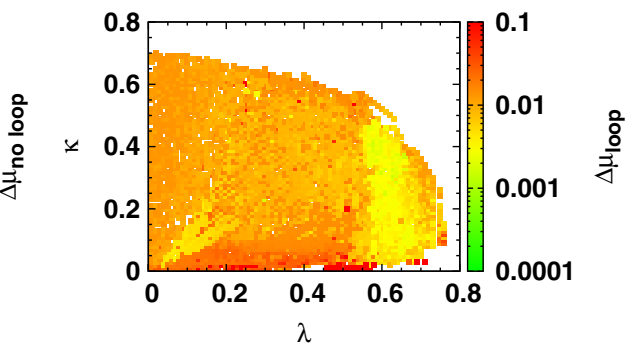

(d)

FIG. 9. The total $\chi^{2}$ function in the $\lambda-\kappa$ plane, as shown by the color coding in (a). The two green regions with the smallest $\chi^{2}$ values are called eegion I (II) for the region on the right (left). Here, all constraints are fulfilled. Note that the absolute $\chi^{2}$ value depends on the choice of the error for the selected Higgs mass combination, but the minimum of the $\chi^{2}$ distribution is always in the green regions. Different errors only shift the intensity of the shading. For small values of $\lambda$ and large values of $\kappa$, the constraints are not fulfilled by the Higgs mass, since the Higgs mass is too low in this region, as shown by the shading in (b). In addition, the fitted signal strengths $\mu_{\text {no-loop }}$ deviates from 1 as can be seen from (c), where the averaged deviations from the SM value are shown. For intermediate values of $\lambda$ and $\kappa$, the main contribution to the total $\chi^{2}$ function is coming from the fermion signal strengths $\mu_{\text {no-loop. }}$. The remaining signal strengths $\mu_{\text {loop }}$, shown by the averaged difference from the SM value (d), deviates from 1 in almost the whole $\lambda-\kappa$ plane, except for region I as shown in (d) by the averaged deviations from the SM value. 


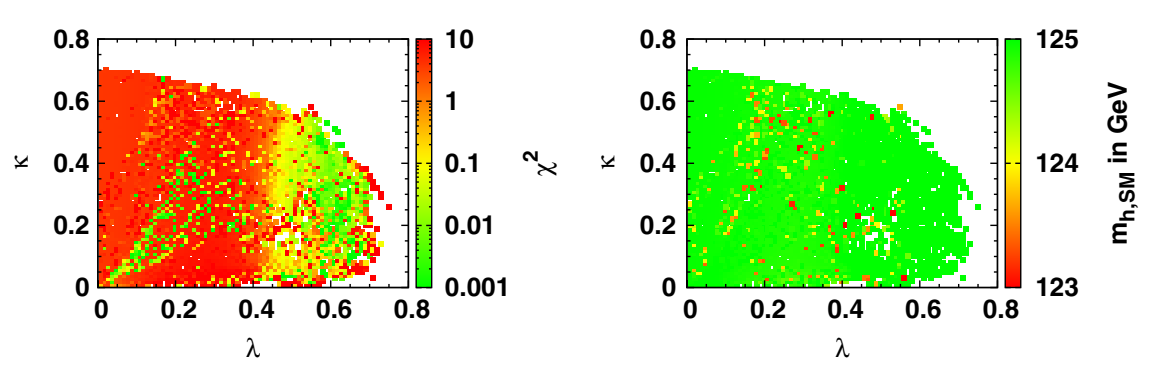

(a)

(b)

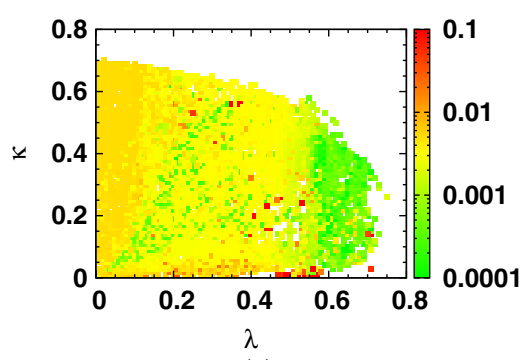

(c)

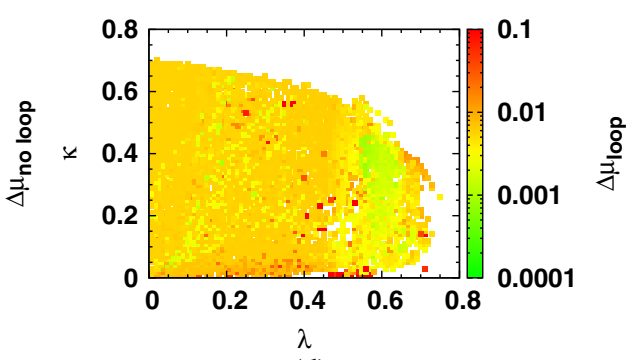

(d)

FIG. 10. As Fig. 9, but for $m_{0}=m_{1 / 2}=1.5 \mathrm{TeV}$.

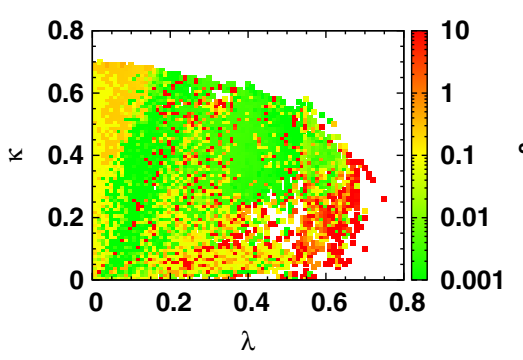

(a)

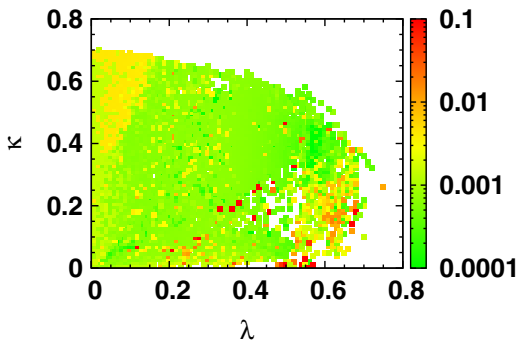

(c)

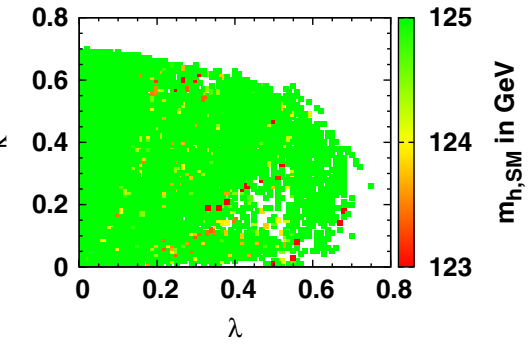

(b)

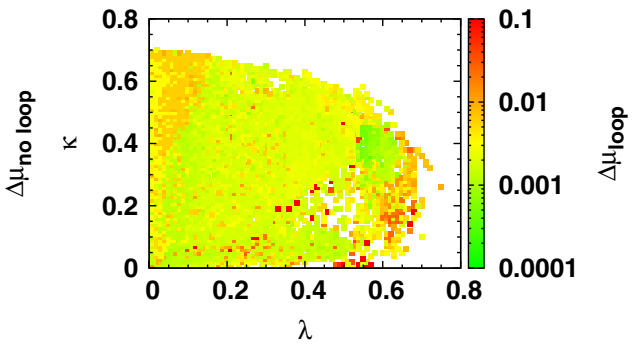

(d)

FIG. 11. As Fig. 9, but for $m_{0}=m_{1 / 2}=2 \mathrm{TeV}$.

the $\chi^{2}$ function for a given combination of $\lambda$ and $\kappa$. The differences with the standard fit procedure, as discussed in Sec. III, have been summarized in Table I.

The two regions with the smallest $\chi^{2}$ values in the $\lambda-\kappa$ plane are the light shaded ( green) regions in Fig. 9(a). The remaining regions-called intermediate regions-have a larger $\chi^{2}$ value for various reasons: for small values of $\lambda$ and large values of $\kappa$, the Higgs mass of the observed Higgs boson is too low, as can be seen from Fig. 9(b), where the color coding corresponds to the Higgs boson mass. The low Higgs mass (around $123 \mathrm{GeV}$ ) originates from the fact that the stop corrections are too low for the chosen values of $m_{0}=m_{1 / 2}=1 \mathrm{TeV}$. In addition, the fitted signal strength $\mu_{\text {no-loop }}$ deviates from 1 as can be seen from Fig. 9(c), where the color coding corresponds to the averaged difference from the $\mathrm{SM}$ value, i.e., $\Delta \mu_{\text {no-loop }}=\frac{1}{4} \sum_{i=1}^{4}\left(\mu^{i}-\mu_{\text {theo }}\right)^{2} / \sigma_{\mu}^{2}$ with $\mu^{i}=\mu_{\tau \tau}^{V B F / V H}, \mu_{b b}^{t t H}, \mu_{b b}^{V B F / V H}$ and $\mu_{Z Z / W W}^{V B F / V H}$. The averaged deviations of the remaining signal strengths $\mu_{\text {loop }}$, i.e., $\Delta \mu_{\text {loop }}=\frac{1}{4} \sum_{i=1}^{4}\left(\mu^{i}-\mu_{\text {theo }}\right)^{2} / \sigma_{\mu}^{2}$ where $\mu_{i}$ includes $\mu_{\tau \tau}^{g g f}$, $\mu_{Z Z / W W}^{g g f}, \mu_{\gamma \gamma}^{V B F / V H}$ and $\mu_{\gamma \gamma}^{g g f}$ is shown in Fig. 9(d). Here, the deviations of the signal strengths $\mu_{\text {loop }}$ of the order of a few percent correspond to the orange region. 
If one increases the $m_{0}=m_{1 / 2}$ values from $1 \mathrm{TeV}$ in Fig. 9 to 1.5 (2) TeV in Figs. 10 (11), one observes that the $\chi^{2}$ values in panels (a) decrease, mainly because of the increased values of the Higgs mass [panels (b)] and the decrease in the deviations of the signal strengths from the SM expectations [panels (c) and (d)]. Note that the absolute values of the test statistic $\chi^{2}$ are somewhat arbitrary because of the choice of the errors for the assumed Higgs mass values of the three chosen Higgs masses on the grid in Fig. 2, i.e., $m_{H_{1}}, m_{A_{1}}$, and $m_{H_{3}}$. But the regions with minimum $\chi^{2}$ values stay the same, as do the deviations of the signal strengths from the SM expectations.
[1] D. Miller, R. Nevzorov, and P. Zerwas, The Higgs sector of the next-to-minimal supersymmetric standard model, Nucl. Phys. B681, 3 (2004).

[2] U. Ellwanger, C. Hugonie, and A. M. Teixeira, The next-tominimal supersymmetric Standard Model, Phys. Rep. 496, 1 (2010).

[3] ATLAS Collaboration, Observation of a new particle in the search for the Standard Model Higgs boson with the ATLAS detector at the LHC, Phys. Lett. B 716, 1 (2012).

[4] CMS Collaboration, Observation of a new boson at a mass of $125 \mathrm{GeV}$ with the CMS experiment at the LHC, Phys. Lett. B 716, 30 (2012).

[5] C. Beskidt, W. de Boer, D. Kazakov, and F. Ratnikov, Constraints on supersymmetry from LHC data on SUSY searches and Higgs bosons combined with cosmology and direct dark matter searches, Eur. Phys. J. C 72, 2166 (2012).

[6] O. Buchmueller, R. Cavanaugh, A. De Roeck et al., The CMSSM and NUHM1 after LHC Run 1, Eur. Phys. J. C 74, 2922 (2014).

[7] A. Fowlie, M. Kazana, K. Kowalska, S. Munir, L. Roszkowski, E. M. Sessolo, S. Trojanowski, and Y.-L. Sming Tsai, The CMSSM favoring new territories: The impact of new LHC limits and a $125 \mathrm{GeV}$ Higgs, Phys. Rev. D 86, 075010 (2012).

[8] P. Bechtle, K. Desch, H. K. Dreiner et al., Constrained supersymmetry after the Higgs boson discovery: A global analysis with Fittino, Proc. Sci., EPS-HEP2013 (2013) 313.

[9] C. Beskidt, W. de Boer, and D. Kazakov, A comparison of the Higgs sectors of the CMSSM and NMSSM for a 126 GeV Higgs boson, Phys. Lett. B 726, 758 (2013).

[10] H. E. Haber and G. L. Kane, The search for supersymmetry: Probing physics beyond the Standard Model, Phys. Rep. 117, 75 (1985).

[11] W. de Boer, Grand unified theories and supersymmetry in particle physics and cosmology, Prog. Part. Nucl. Phys. 33, 201 (1994).

[12] S. P. Martin, A Supersymmetry primer, Adv. Ser. Dir. High Energy Phys. 18, 1 (1998).

[13] C. Beskidt, W. de Boer, D. I. Kazakov, and S. Wayand, Perspectives of direct detection of supersymmetric dark matter in the NMSSM, Phys. Lett. B 771, 611 (2017).

[14] R. Dermisek and J. F. Gunion, Many Light Higgs Bosons in the NMSSM, Phys. Rev. D 79, 055014 (2009).

[15] S. King, M. Mühlleitner, and R. Nevzorov, NMSSM Higgs benchmarks near $125 \mathrm{GeV}$, Nucl. Phys. B860, 207 (2012).

[16] J.-J. Cao, Z.-X. Heng, J. M. Yang, Y. Zhang, and J. Zhu, A SM-like Higgs near $125 \mathrm{GeV}$ in low energy
SUSY: A comparative study for MSSM and NMSSM, J. High Energy Phys. 03 (2012) 086.

[17] U. Ellwanger and C. Hugonie, Higgs bosons near $125 \mathrm{GeV}$ in the NMSSM with constraints at the GUT scale, Adv. High Energy Phys. 2012, 1 (2012).

[18] J. F. Gunion, Y. Jiang, and S. Kraml, The constrained NMSSM and Higgs near $125 \mathrm{GeV}$, Phys. Lett. B 710, 454 (2012).

[19] J. Cao, F. Ding, C. Han, J. M. Yang, and J. Zhu, A light Higgs scalar in the NMSSM confronted with the latest LHC Higgs data, J. High Energy Phys. 11 (2013) 018.

[20] M. Badziak, M. Olechowski, and S. Pokorski, New regions in the NMSSM with a $125 \mathrm{GeV}$ Higgs, J. High Energy Phys. 06 (2013) 043.

[21] R. Barbieri, D. Buttazzo, K. Kannike, F. Sala, and A. Tesi, One or more Higgs bosons, Phys. Rev. D 88, 055011 (2013).

[22] S. F. King, M. Mühlleitner, R. Nevzorov, and K. Walz, Discovery prospects for NMSSM Higgs bosons at the highenergy large hadron collider, Phys. Rev. D 90, 095014 (2014).

[23] J. Bernon, J. F. Gunion, Y. Jiang, and S. Kraml, Light Higgs bosons in two-Higgs-doublet models, Phys. Rev. D 91, 075019 (2015).

[24] M. Guchait and J. Kumar, Light Higgs bosons in NMSSM at the LHC, Int. J. Mod. Phys. A 31, 1650069 (2016).

[25] C. T. Potter, Natural NMSSM with a Light Singlet Higgs and Singlino LSP, Eur. Phys. J. C 76, 44 (2016).

[26] P. Bandyopadhyay, C. Coriano, and A. Costantini, Probing the hidden Higgs bosons of the $Y=0$ triplet- and singletextended supersymmetric Standard Model at the LHC, J. High Energy Phys. 12 (2015) 127.

[27] N.-E. Bomark, S. Moretti, S. Munir et al., A light NMSSM pseudoscalar Higgs boson at the LHC Run 2, edited by S Kanemura, in 2nd Toyama International Workshop on Higgs as a Probe of New Physics (HPNP2015) Toyama, Japan, 2015 (Univ. of Toyama, Toyama, 2015), https:// www.slac.stanford.edu/econf/C130213.1/.

[28] J. Cao, X. Guo, Y. He, and Y. Zhang, Diphoton signal of the light Higgs boson in natural NMSSM, Phys. Rev. D 95, 116001 (2017).

[29] M. Mühlleitner, M. O. P. Sampaio, R. Santos, and J. Wittbrodt, Phenomenological comparison of models with extended Higgs sectors, J. High Energy Phys. 08 (2017) 132.

[30] S. P. Das and M. Nowakowski, Light neutral $C P$-even Higgs boson within next-to-minimal supersymmetric Standard 
Model (NMSSM) at the large hadron electron collider (LHeC), Phys. Rev. D 96, 055014 (2017).

[31] A. Mariotti, D. Redigolo, F. Sala, and K. Tobioka, New LHC bound on low-mass diphoton resonances, Phys. Lett. B 783, 13 (2018).

[32] S. Baum, K. Freese, N. R. Shah, and B. Shakya, NMSSM Higgs boson search strategies at the LHC and the mono-Higgs signature in particular, Phys. Rev. D 95, 115036 (2017).

[33] C. Beskidt, W. de Boer, D. I. Kazakov, and S. Wayand, Higgs branching ratios in constrained minimal and next-tominimal supersymmetry scenarios surveyed, Phys. Lett. B 759, 141 (2016).

[34] C. Beskidt, W. de Boer, and D. I. Kazakov, Can we discover a light singlet-like NMSSM Higgs boson at the LHC, Phys. Lett. B 782, 69 (2018).

[35] D. Das, U. Ellwanger, and A. M. Teixeira, NMSDECAY: A Fortran code for supersymmetric particle decays in the next-to-minimal supersymmetric Standard Model, Comput. Phys. Commun. 183, 774 (2012).

[36] ATLAS Collaboration, Summary of the searches for squarks and gluinos using $\sqrt{s}=8 \mathrm{TeV} p$ collisions with the ATLAS experiment at the LHC, J. High Energy Phys. 10 (2015) 054.

[37] A. Djouadi, The anatomy of electro-weak symmetry breaking. I: The Higgs boson in the Standard Model, Phys. Rep. 457, 1 (2008).
[38] H. E. Haber, Challenges for nonminimal Higgs searches at future colliders, in Perspectives for electroweak interactions in $e+e-$ collisions, in Proceedings of the Ringberg Workshop, Tegernsee, Germany, 1995, edited by B. A. Kniehl and N. J. River Edge (World Scientific, Singapore, 1996), pp. 219-232.

[39] A. Djouadi, The anatomy of electro-weak symmetry breaking. II. The Higgs bosons in the minimal supersymmetric model, Phys. Rep. 459, 1 (2008).

[40] F. James and M. Roos, Minuit: A system for function minimization and analysis of the parameter errors and correlations, Comput. Phys. Commun. 10, 343 (1975).

[41] C. Beskidt, Supersymmetry in the light of dark matter and a $125 \mathrm{GeV}$ Higgs boson. Ph.D. thesis, KIT, Karlsruhe, EKP, 2014.

[42] CMS Collaboration, Search for a very light NMSSM Higgs boson produced in decays of the $125 \mathrm{GeV}$ scalar boson and decaying into $\tau$ leptons in pp collisions at $\sqrt{s}=8 \mathrm{TeV}$, J. High Energy Phys. 01 (2016) 079.

[43] ATLAS Collaboration, Search for Higgs bosons decaying to $a a$ in the $\mu \mu \tau \tau$ final state in $p p$ collisions at $\sqrt{s}=8 \mathrm{TeV}$ with the ATLAS experiment, Phys. Rev. D 92, 052002 (2015).

[44] CMS Collaboration, A search for pair production of new light bosons decaying into muons, Phys. Lett. B 752, 146 (2016). 